\title{
Análise do comportamento mecânico ao incorporar parafina em misturas EPDM/PP
}

\author{
Mechanical behavior analysis by incorporating paraffin in \\ EPDM/PP mixtures
}

\author{
Eduardo Filipetto Martins ${ }^{1}$ \\ Cristiane Krause Santin ${ }^{2}$
}

\begin{abstract}
Resumo
Neste estudo, a versatilidade dos materiais elastoméricos termoplásticos vulcanizados (TPVs) foi explorada, ao combinar as características da mistura EPDM/PP, ao avaliar a influência provocada em suas propriedades mecânicas e ao inserir parafina na composição do material, visando futura atuação como material de troca de fase (PCM), por meio de análises de tempo de cura, resistência à tração e dureza Shore A. Além disso, as suas características estruturais e morfológicas foram observadas, através de análises por infravermelho e microscopia eletrônica de varredura $(\mathrm{MEV})$. O comportamento de inchamento complementou a análise, quanto à influência da parafina inserida no material final. A partir dos testes mecânicos, evidenciou-se que o gradual acréscimo de parafina na composição da mistura EPDM/PP ocasionou uma pequena diminuição, tanto na tensão de ruptura do material, quanto no seu alongamento. Ainda, através da análise por MEV, observou-se uma distribuição superficial uniforme da parafina sobre o material TPV desenvolvido.
\end{abstract}

Palavras-chave: EPDM/PP. Parafina. Propriedades mecânicas.

\begin{abstract}
In this study, the versatility of vulcanized thermoplastic elastomeric materials (TPVs) was explored by combining the characteristics of the EPDM/PP mixture, by evaluating the influence caused in its mechanical properties and by inserting paraffin in the composition, aiming at future performance as phase change material (PCM), through analysis on cure time, tensile strength and Shore A hardness. Moreover, its structural and morphological characteristics were observed through infrared analysis and scanning electron microscopy (SEM). The swelling behavior complemented the analysis, regarding the influence of paraffin inserted in the final material. From the mechanical tests, it was evidenced that the gradual addition of paraffin in the composition of the EPDM/PP mixture caused a small decrease in both at the material rupture tension, and in its elongation. Furthermore, through SEM analysis, it was observed a uniform surface distribution of paraffin on the developed TPV material.
\end{abstract}

Keywords: EPDM/PP. Paraffin. Mechanical properties.

1 Bacharel em Engenharia Química pela Universidade do Vale do Rio dos Sinos (UNISINOS), São Leopoldo, RS, Brasil. ORCID: https:// orcid.org/ 0000-0002-3428-924X. E-mail: eduardo.filipetto@gmail.com

2 Doutora em Ciência dos Materiais pela Universidade Federal do Rio Grande do Sul (UFRGS), Porto Alegre, RS e mestre em Química pela UFRGS. Professora na UNISINOS. ORCID: https://orcid.org/ 0000-0002-9683-678X. E-mail: cksantin@unisinos.br

Artigo recebido em 21.09.2021 e aceito em 16.11.2021. 


\section{Introdução}

Os elastômeros termoplásticos vulcanizados (TPVs) são amplamente utilizados numa variedade de aplicações de uso comum que vão desde dispositivos médicos, componentes e peças de automóveis, bem como revestimentos (NING et al., 2018). Apresentam em sua composição dois ou mais componentes poliméricos, formando uma mistura com características e propriedades diferenciadas. Por serem constituídos por mais de um material polimérico, possuem uma morfologia composta por uma fase contínua e, outra dispersa, evidenciadas por análises microscópicas.

Nos últimos anos, numerosos estudos têm sido realizados para o desenvolvimento de elastômeros termoplásticos (TPEs) e elastômeros termoplásticos dinamicamente vulcanizados (TPVs), assim como, para compreensão de suas características e potencialidades (CHO et al., 2017; HOLDEN; KRICHELDORF; QUIRK, 2004; IMATO et al., 2016; MATEI et al., 2017; MCMULLIN; REBAR; MATHER, 2016; YU et al., 2020).

Os elastômeros termoplásticos e os elastômeros termoplásticos, dinamicamente vulcanizados, baseados em polipropileno (PP)/etileno-propileno dieno (EPDM), demonstram boas características de resistência ao calor, ao óleo e ao comportamento mecânico e também são comprovados pela sua ampla utilização na indústria de automóveis, na construção e em aplicações domésticas (WATTS; KUROKAWA; HILLMYER, 2017).

Nas últimas décadas, o interesse por fontes renováveis de energia tornou-se mais recorrente, devido à escassez de recursos naturais, diversificação da matriz energética e busca por fontes de energia mais sustentáveis. A partir disso, novas possibilidades de materiais, com a finalidade de melhorar a eficiência do uso desse recurso finito, tornam-se indispensáveis. A partir disso, foram investigados nos estudos de Gulfam, Zhang e Meng (2019) e Luo et al. (2017) sistemas solares térmicos, utilizando PCMs (Phase Change Material) em painéis fotovoltaicos, para tecnologia de geração de energia.

Os materiais PCMs caracterizam-se por apresentar um alto valor quanto ao calor latente, cuja propriedade lhes confere uma atuação como armazenadores de energia, cuja característica pode ser monitorada por uma variação na entalpia, durante a transição de fase física do material. Ainda, o interesse pelo desenvolvimento de materiais, com potencial atuação PCMs, deve-se a sua elevada energia, densidade de armazenamento e a possibilidade de aplicação em faixas limitadas de temperatura (RAMAKRISHNAN et al., 2015). O calor latente e a temperatura são os parâmetros mais importantes a considerar, para a utilização de PCM, em qualquer processo (KUMAR; JEGADHEESWARAN; CHANDRAMOHAN, 2018). Estudos realizados por Banno et al. (2019) e Boiocchi et al. (2016) exploraram o uso de PCMs na combinação de combustíveis à base de parafina com outros polímeros termoplásticos, para a síntese de combustível espacial, onde os termoplásticos inseridos atuariam para prolongar o tempo de queima, sem aumento de volume. Opções para fontes renováveis de energia também foram estudadas por Chen et al. (2016) com espuma metálica embutida em SEBS/parafina/PEAD, analisando as amostras obtidas quanto a sua morfologia e quantificando o calor fornecido.

No processo de vulcanização dinâmica a mistura entre os componentes presentes e o processo de reticulação ocorrem simultaneamente, associadas a uma elevada taxa de cisalhamento e temperatura elevada de mistura (CHATTERJEE et al., 2014), obtendo-se um produto com predomínio de propriedades, características de um material elástico, como por exemplo, compressão e rigidez mais baixas, quando comparadas com as obtidas para os materiais individualmente analisados (CORAN, 1995). A elevada viscosidade do elastômero influencia a morfologia da mistura elastômero-termoplástico, promovendo um fenômeno de inversão de fase, ou seja, a mudança, onde a fase contínua torna-se a fase dispersa e vice-versa (ELLUL; TSOU; HU, 2004). O processo, para obtenção do material TPV, contempla a etapa de introdução dos polímeros na câmara de mistura ou no processo de extrusão, com posterior inserção do sistema de cura, seguido do processo de reticulação a uma temperatura pré-determinada e, por fim, o processo de conformação como, por exemplo, injeção (CORAN, 1995).

O objetivo deste trabalho visa analisar o comportamento mecânico, morfológico e de expansão em solvente de uma mistura de EPDM/PP ao inserir parafina, visando sua atuação e futura aplicação como PCM, assim como estabelecer a etapa ideal de inserção e percentuais de parafina que promovam melhores características para um material versátil e com potencialidade para recobrimento de superfícies de equipamentos térmicos, tendo como finalidade maior eficiência térmica de processo. 


\section{Desenvolvimento}

\subsection{Materiais}

Os materiais utilizados neste estudo foram: EPDM Keltan 312, com 50\% em massa de etileno e $4 \%$ em massa de etilideno norborneno (ENB), com distribuição de massa molecular média e uma viscosidade Mooney ML $(1+4) 36$ a $125^{\circ} \mathrm{C}$, fornecida pela Lanxess Deutschland $\mathrm{GmbH}$, Cologne, Alemanha, como fase elastoméricas e, como componente plástico da mistura, o homopolímero de polipropileno isotático $\left(\mathrm{PP}_{\text {homo }}\right)$ Moplen HP500N, fornecido pela empresa LyondellBasel, Frankfurt, Germany.

O sistema de cura utilizado foi: Di(2-tercbutilperoxi-isopropil) benzeno (Perkadox 1440B) da empresa Pergan Gmbh, Bocholt, Alemanha, e cianurato de trialil (TAC50) da empresa Lanxess Deutschland $\mathrm{GmbH}$, Cologne, Alemanha como co-agente.

A parafina utilizada neste estudo com a composição 1-metoxi-2-propanol (8\%), metiletilcetona (76\%) e xileno (16\%), com um ponto de fusão entre $58^{\circ} \mathrm{C}$ e $60^{\circ} \mathrm{C}$, foi proveniente da empresa Grüssing $\mathrm{GmbH}$, Filsum, Alemanha.

\subsection{Condição do processo e preparação da mistura}

A mistura foi obtida em processo descontínuo, utilizando um misturador interno Haake PolylabTM QC modulares Drehmoment-Rheometer com Rotores Banbury R600, contendo um volume completo de $78 \mathrm{~cm}^{3}$. A velocidade de rotação para a primeira batelada foi de $40 \mathrm{rpm}$, enquanto para a segunda batelada, foi de $100 \mathrm{rpm}$. Para obter a mistura (TPV e, posteriormente, TPV + parafina), foi aplicada uma temperatura de 100 a $170{ }^{\circ} \mathrm{C}$, com um fator de enchimento da câmara de 0,8 , ou seja, $80 \%$.
A composição da mistura $\mathrm{EPDM} / \mathrm{PP}_{\text {homo }}$ utilizada foi de 100/30 phr respectivamente, demonstrando a proporção com um total de 100 partes.

A mistura foi obtida em duas etapas: primeira batelada e segunda batelada. As condições de processo no equipamento misturador Haake, bem como: o EPDM, $\mathrm{PP}_{\text {homo }}$ e a concentração da pré-batelada estão descritas na figura 1 . As quantidades de agente de cura e de co-cura variaram entre 0,5 a 3,0 phr e, para a parafina, variou entre 5 a $30 \mathrm{phr}$.

As amostras de referência denominadas EPDM Puro, EPDM + $\mathrm{PP}_{\text {homo }}$ e EPDM + Parafina foram obtidas sob as mesmas condições de processo cuja formulação é mostrada na tabela 1 . O procedimento, para a obtenção das amostras de referência, seguiu o demonstrado no esquema, figura 1.

Figura 1 - Esquema do processo para a obtenção do produto TPV

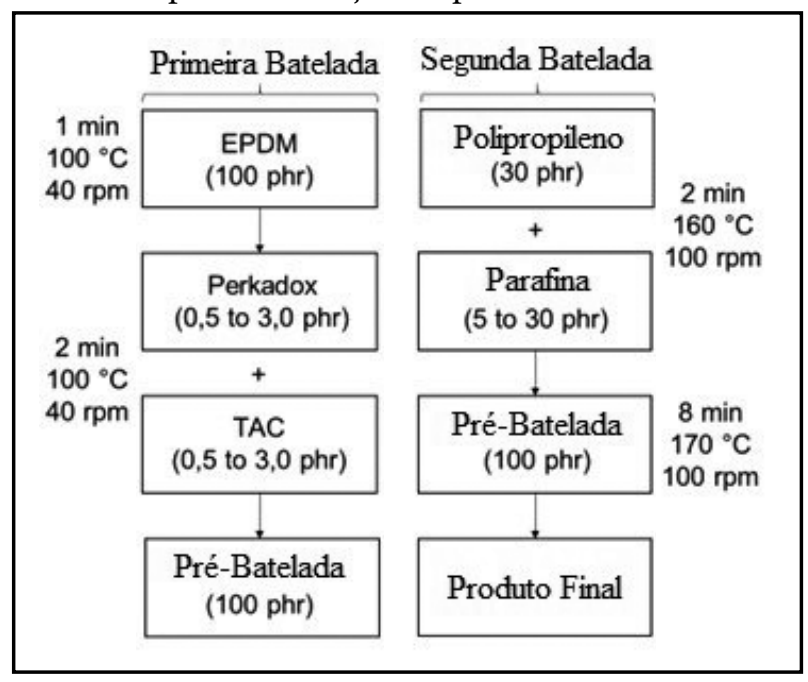

Fonte: Os autores (2019).

Tabela 1- Composição (phr) das misturas de referência

\begin{tabular}{cccccc}
\hline Amostra & $\begin{array}{c}\text { EPDM } \\
(\mathbf{p h r})\end{array}$ & $\begin{array}{c}\text { Perkadox } \\
\mathbf{1 4 4 0 B}(\mathbf{p h r})\end{array}$ & $\begin{array}{c}\text { TAC 50 } \\
(\mathbf{p h r})\end{array}$ & $\begin{array}{c}\text { Polipropile- } \\
\text { no (phr) }\end{array}$ & $\begin{array}{c}\text { Parafina } \\
\text { (phr) }\end{array}$ \\
\hline EPDM Puro & 100 & - & - & - & - \\
EPDM + PP & 100 & - & - & 30 & - \\
EPDM + Parafina & 100 & - & - & - & 30 \\
\hline
\end{tabular}

Fonte: Os autores (2019).

Para o estudo do sistema de cura do TPV, foram produzidas seis misturas $\mathrm{EPDM} / \mathrm{PP}_{\text {homo }}$, nas quais a proporção do sistema de cura Perkadox/TAC variou entre 0,5-3,0 phr, tabela 2.

$O$ estudo da etapa de adição da parafina foi realizado nas seguintes condições: Amostra 1, durante o processamento pré-batelada (EPDM + sistema de cura + parafina a $100^{\circ} \mathrm{C}$ ), Amostra 2, após a conclusão do pré-batelada, estudando a inserção da parafina antes do processo de reticulação (parafina $+\mathrm{PP}_{\text {homo }}$ a $160^{\circ} \mathrm{C}$ ) e, Amostra 3, após o processo de reticulação do elastômero $\left(\mathrm{PP}_{\text {homo }}+\right.$ parafina a $\left.170^{\circ} \mathrm{C}\right)$, onde a parafina foi o último componente a ser incorporado na mistura.

Ainda, foram analisadas diferentes formulações, tabela 3, para investigar a influência da parafina nas propriedades do produto acabado. 
Tabela 2 - Formulação das amostras para o estudo do sistema de cura

\begin{tabular}{cccc}
\hline $\begin{array}{c}\text { Amostra } \\
\text { (Perkadox/TAC } \mathbf{p h r})\end{array}$ & $\begin{array}{c}\text { EPDM } \\
\text { (phr) }\end{array}$ & $\begin{array}{c}\text { Perkadox } \\
\text { 1440B (phr) }\end{array}$ & $\begin{array}{c}\text { TAC50 } \\
\text { (phr) }\end{array}$ \\
\hline 0,5 & 100 & 2,5 & 2,5 \\
1,0 & 100 & 2,5 & 2,5 \\
1,5 & 100 & 2,5 & 2,5 \\
2,0 & 100 & 2,5 & 2,5 \\
2,5 & 100 & 2,5 & 2,5 \\
3,0 & 100 & 2,5 & 2,5 \\
\hline
\end{tabular}

Fonte: Os autores (2019).

Tabela 3 - Formulação das amostras para o estudo da parafina

\begin{tabular}{|c|c|c|c|c|c|}
\hline Amostra & $\begin{array}{c}\text { EPDM } \\
\text { (phr) }\end{array}$ & $\begin{array}{l}\text { Perkadox } \\
\text { 1440B (phr) }\end{array}$ & $\begin{array}{c}\text { TAC50 } \\
\text { (phr) }\end{array}$ & $\begin{array}{c}\text { Pré-batelada }^{1} \\
\text { (phr) }\end{array}$ & $\begin{array}{c}\text { Parafina } \\
\text { (phr) }\end{array}$ \\
\hline $5 \mathrm{phr}$ & 100 & 2,5 & 2,5 & 100 & 5 \\
\hline $10 \mathrm{phr}$ & 100 & 2,5 & 2,5 & 100 & 10 \\
\hline $15 \mathrm{phr}$ & 100 & 2,5 & 2,5 & 100 & 15 \\
\hline $20 \mathrm{phr}$ & 100 & 2,5 & 2,5 & 100 & 20 \\
\hline $25 \mathrm{phr}$ & 100 & 2,5 & 2,5 & 100 & 25 \\
\hline $30 \mathrm{phr}$ & 100 & 2,5 & 2,5 & 100 & 30 \\
\hline
\end{tabular}

${ }^{1}$ Pré-batelada $=$ EPDM + Perkadox 1440B + TAC 50

Fonte: Os autores (2019).

\subsection{Caracterização}

Para os ensaios de caracterização mecânica, foram obtidas placas de ensaio de $2 \mathrm{~mm}$ de espessura, a partir do processo de injeção e moldagem. As amostras foram inseridas no sistema de moldagem por injeção da Testing Machines Inc. com uma configuração de pré-aquecimento de $190^{\circ} \mathrm{C}$.

Para obter o tempo e temperatura de cura ótimos, as misturas foram submetidas à análise em reômetro dinâmico - móvel D-MDR 3000, contendo agentes de cura sob condições de $160^{\circ} \mathrm{C}$, durante 20 minutos e frequência de $1 \mathrm{~Hz}$.

Os ensaios mecânicos de tração foram realizados, utilizando uma máquina de ensaio Zwick Materials 1465 , usando configurações para $1 \mathrm{kN}$ de força nominal, $1 \mathrm{~N}$ de força de avanço e com um dispositivo pneumático de fixação, atuando como suporte de amostras. O ensaio foi realizado em quintuplicata para obter uma média aritmética dos valores da resistência à tração e ao alongamento na ruptura. $\mathrm{O}$ ensaio de dureza foi realizado com um Durômetro Bareiss Shore A, baseado na ASTM D 2240.
A morfologia da mistura obtida foi avaliada por microscópio eletrônico de varredura (MEV) TESCAN VEGA3 LMU. Realizou-se a quebra criogênica das amostras, com posterior extração da fase termoplástica com solvente xileno, seguida de evaporação e secagem. Posteriormente, as amostras foram metalizadas por pulverização com ouro e, em seguida, foi executado o teste de microscopia eletrônica de varredura de elétrons secundários com $15 \mathrm{kV}$ de voltagem aplicada e magnificação de 4000 vezes. Os resíduos da extração da fase termoplástica com solvente xileno foram analisados por Espectroscopia de Infravermelho por Transformada de Fourier com Refletânica Total Atenuada no equipamento Carry 630, Agilent Technologies. A análise foi realizada entre a região de $700 \mathrm{~cm}^{-1}$ a $4000 \mathrm{~cm}^{-1}$ com 32 varreduras, observando as bandas na região de $2840 \mathrm{~cm}^{-1}, 3000 \mathrm{~cm}^{-1}$, e 1450 $\mathrm{cm}^{-1}$, características dos grupos $-\mathrm{CH}_{3} \mathrm{e}-\mathrm{CH}_{2}$.

Para avaliar o comportamento com solvente e com processo de expansão, as amostras foram submetidas ao teste de inchamento com solvente ciclohexano a uma temperatura de $23^{\circ} \mathrm{C}$, sob banho termostatizado. 
Os testes foram realizados em duplicada, obtendo-se o valor da massa para cálculo pela média aritmética.

As amostras foram inseridas no sistema, contendo o solvente ciclohexano em equilíbrio térmico, até se atingir uma massa constante. Posteriormente, a amostra foi seca numa estufa a $40^{\circ} \mathrm{C}$. O grau de inchamento ou expansão da amostra foi obtido, através da equação 1, e a fração volumétrica do polímero " $\mathrm{Vr}$ ", na amostra inchada, foi obtida, através da equação 2.

$$
\begin{gathered}
\mathrm{Q}=(\text { massa de solvente absorvida }(\mathrm{g})) /(\text { massa inicial }(\mathrm{g})) \\
\qquad \mathrm{Vr}=1 / \mathrm{Q},
\end{gathered}
$$

\section{Resultados e discussão}

\subsection{Cura}

A figura 2 mostra o torque versus tempo para amostras da pré-batelada, com diferentes quantidades de sistema de cura (em phr) de Perkadox/ TAC50 a $170{ }^{\circ} \mathrm{C}$.

Nos resultados, demonstrados na figura 2, foi observado um aumento do torque com o tempo em todas as amostras, na faixa de aproximadamente
1300 segundos, é identificado um comportamento de estabilização constante do torque, em relação ao tempo. Esse ponto é chamado $t_{90}$ que, de acordo com a literatura, corresponde a $90 \%$ de cura do elastômero (KHIMI; PICKERING, 2014), o qual é obtido, a partir da equação 3:

$$
\mathrm{X}(\mathrm{t})=(\mathrm{F}(\mathrm{t})-\mathrm{Fmin}) /(\mathrm{Fmax}-\mathrm{Fmin}) \times 100 \% \text {, }
$$

onde, $\mathrm{X}$ é a conversão dada em \%, $\mathrm{F}(\mathrm{t})$ é o torque medido no momento t, Fmin e Fmax são o torque mínimo e máximo, respectivamente.

Figura 2 - Curva de torque para amostras de pré-batelada, contendo 0,5 phr a 3,0 $\mathrm{phr}$ de Perkadox/TAC50

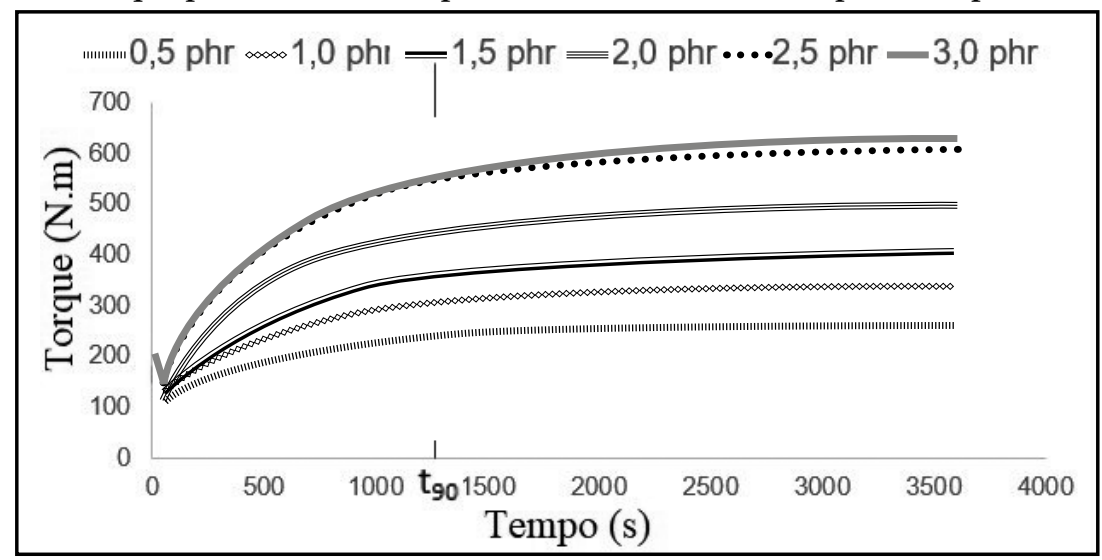

Fonte: Os autores (2019).

O aumento da quantidade de agentes de cura promove a formação de mais ligações cruzadas entre cadeias, o que se observa no aumento do torque evidenciado. As amostras com 2,5 e 3,0 phr de Perkadox/ TAC50 mostraram um comportamento muito semelhante, com um ponto de saturação ou limite máximo do processo de reticulação para a condição utilizada.

\subsection{Ensaios mecânicos}

Os materiais de referência EPDM puro, EPDM $+\mathrm{PP}_{\text {homo }} \mathrm{e} \mathrm{EPDM}+$ Parafina foram submetidos a ensaios de tração, para posterior comparação com as misturas desenvolvidas. A tabela 4 apresenta os resultados obtidos.

Tabela 4 - Resultados da resistência à tração, ao alongamento, ao módulo de Young e à dureza Shore A, para as amostras de referência

\begin{tabular}{ccccc}
\hline Amostra & $\begin{array}{c}\text { Tensão de } \\
\text { ruptura (MPa) }\end{array}$ & $\begin{array}{c}\text { Alongamento } \\
\text { na ruptura (\%) }\end{array}$ & $\begin{array}{c}\text { Módulo de } \\
\text { Young (MPa) }\end{array}$ & $\begin{array}{c}\text { Dureza } \\
\text { Shore A }\end{array}$ \\
\hline EPDM Puro & $1,1 \pm 0,0$ & $945,3 \pm 23,2$ & 0,02 & $21,3 \pm 0,8$ \\
EPDM + PP & $3,5 \pm 0,2$ & $481,1 \pm 36,1$ & 0,11 & $64,8 \pm 1,6$ \\
EPDM + Parafina & $5,5 \pm 0,7$ & $884,0 \pm 18,8$ & 0,10 & $51,0 \pm 3,0$ \\
\hline
\end{tabular}

Fonte: Os autores (2019). 
A partir dos resultados da tabela 4, observa-se um aumento da resistência à tração e à dureza Shore $\mathrm{A}$ da amostra EPDM + $\mathrm{PP}_{\text {homo }}$, em relação ao EPDM Puro, assim como a diminuição dos resultados do alongamento na ruptura, para a amostra ao ser comparada com o elastômero puro. Esse comportamento pode estar relacionado com a estrutura e com as características químicas de $\mathrm{PP}_{\text {homo, }}$ presentes na composição que influenciaram o movimento das ligações químicas e, consequentemente, as propriedades físicas e mecânicas da amostra, contendo o componente plástico.

A amostra EPDM + Parafina 100/30 demonstrou o módulo de Young muito próximo da mistura $\mathrm{EPDM}+\mathrm{PP}_{\text {homo }}$. Além disso, observou-se um aumento significativo do alongamento na ruptura, fato que pode estar associado a uma redistribuição das aglomerações de carbono, promovido pela presença da parafina, movimentando as cadeias de forma a que elas se distribuam mais uniformemente na estrutura química do EPDM, tornando as zonas amorfas regulares (ABU-ABDEEN, 2001).

\subsection{Composição do sistema de cura}

Os resultados das propriedades mecânicas das misturas EPDM/PP homo $100 / 30 \mathrm{phr}$, com diferentes percentagens do sistema de cura Perkadox/TAC, estão descritos na tabela 5 .

Tabela 5 - Comportamento mecânico das amostras de EPDM/PP ${ }_{\text {homo }} 100 / 30$ phr, com diferentes composições do sistema de cura

\begin{tabular}{ccccc}
\hline $\begin{array}{c}\text { Amostra (Perka- } \\
\text { dox/TAC phr) }\end{array}$ & $\begin{array}{c}\text { Tensão de } \\
\text { ruptura (MPa) }\end{array}$ & $\begin{array}{c}\text { Alongamento } \\
\text { na ruptura (\%) }\end{array}$ & $\begin{array}{c}\text { Módulo de } \\
\text { Young (MPa) }\end{array}$ & Dureza Shore A \\
\hline 0,5 & $4,7 \pm 0,1$ & $490,8 \pm 45,5$ & 0,17 & $75,2 \pm 0,4$ \\
1,0 & $4,5 \pm 0,1$ & $410,7 \pm 10,0$ & 0,19 & $74,2 \pm 1,0$ \\
1,5 & $4,5 \pm 0,2$ & $368,4 \pm 24,5$ & 0,17 & $74,6 \pm 0,5$ \\
2,0 & $4,5 \pm 0,2$ & $328,8 \pm 24,1$ & 0,20 & $76,6 \pm 0,5$ \\
2,5 & $5,5 \pm 0,1$ & $372,7 \pm 9,2$ & 0,18 & $77,5 \pm 0,5$ \\
3,0 & $5,2 \pm 0,4$ & $293,3 \pm 46,4$ & 0,16 & $76,2 \pm 0,4$ \\
\hline
\end{tabular}

Fonte: Os autores (2019).

Sabe-se que o sistema de cura e a sua quantidade utilizada influenciam o grau de reticulação do produto obtido e, consequentemente, as propriedades mecânicas, tais como: alongamento e resistência à tração, ao módulo de Young e à dureza Shore A (NAKASON et al., 2006).

Conforme se observa nos resultados da tabela 5, não ocorre uma alteração significativa na resistência à tração, no módulo Young ou na dureza com o aumento da percentagem do sistema de cura na mistura. No entanto, o alongamento na ruptura mostra uma diminuição de aproximadamente 200\%, quando comparado com o valor mínimo e máximo para a composição do sistema de cura, cujo comportamento pode ser relacionado ao grau de reticulação crescente das amostras, resultando na formação de elos de ligação que, consequentemente aumentam a tensão de ruptura e diminuem a capacidade de alongamento da amostra.

Entre as amostras, salienta-se que a amostra, contendo 2,5 phr do sistema de cura, apresentou um alongamento elevado na ruptura e resistência da tensão de ruptura em comparação com as demais composições utilizadas, cujo comportamento refere-se a um eficiente processo de reticulação do material elastomérico presente, também evidenciado nos resultados de torque obtidos, figura 2 .

\subsection{Incorporação da parafina}

Para o estudo da etapa de incorporação da parafina, foram preparadas três amostras, contendo 10 phr de parafina e inseridas em três momentos do processo: Amostra 2 - parafina na pré-batelada, Amostra 3 - pré-reticulação, Amostra 4- pós-reticulação e, para fins comparativos, a Amostra 1, como referência, não contendo nenhuma parafina em sua composição. A tabela 6 mostra os resultados das propriedades mecânicas, obtidas para as amostras.

Analisando-se a tabela 6, através dos valores de módulo de Young e dureza obtidos, observa-se que a adição de parafina, independente da etapa de sua inserção, não influenciou o parâmetro módulo de Young, assim como a dureza Shore A, cujos valores foram muito próximos entre si. Verifica-se, porém, uma diminuição nos valores de tensão de ruptura e uma variabilidade quanto aos valores do alongamento na ruptura, os quais foram nitidamente observados na incorporação da parafina na etapa de pós-reticulação. 
Tabela 6 - Tensão de ruptura, alongamento na ruptura, módulo de Young, dureza Shore A, para amostras $\mathrm{EPDM} / \mathrm{PP}_{\text {homo }} 100 / 30$, com sistema de cura de 2,5 phr e $10 \mathrm{phr}$ de parafina, adicionadas em diferentes etapas e Amostra $1 \mathrm{EPDM} / \mathrm{PP}_{\text {homo }} 100 / 30$, com sistema de cura de 2,5 phr, sem parafina

\begin{tabular}{ccccc}
\hline $\begin{array}{c}\text { Amostra (Inser- } \\
\text { ção da Parafina) }\end{array}$ & $\begin{array}{c}\text { Tensão de } \\
\text { ruptura (MPa) }\end{array}$ & $\begin{array}{c}\text { Alongamento } \\
\text { na ruptura (\%) }\end{array}$ & $\begin{array}{c}\text { Módulo de } \\
\text { Young (MPa) }\end{array}$ & $\begin{array}{c}\text { Dureza } \\
\text { Shore A }\end{array}$ \\
\hline Amostra 1 & $5,5 \pm 0,1$ & $372,7 \pm 9,2$ & 0,18 & $77,5 \pm 0,5$ \\
Amostra 2 & $5,0 \pm 0,3$ & $294,5 \pm 20,1$ & 0,22 & $80,0 \pm 0,6$ \\
Amostra 3 & $5,0 \pm 0,3$ & $357,3 \pm 24,8$ & 0,21 & $79,5 \pm 1,6$ \\
Amostra 4 & $4,7 \pm 0,2$ & $291,0 \pm 24,1$ & 0,19 & $78,8 \pm 1,2$ \\
\hline
\end{tabular}

Fonte: Os autores (2019).

$\mathrm{Na}$ Amostra 2, a parafina é inserida antes mesmo do elastômero EPDM. Nesse caso, a razão da sua eficiência inferior pode ser a temperatura no misturador interno, pois sendo esta $100^{\circ} \mathrm{C}$, tem-se o derretimento total da parafina, porém essa temperatura não é suficiente para obter a fluidez do EPDM que obtém maior mobilidade de suas cadeias em temperaturas mais altas, dificultando a homogeneização entre a parafina e o elastômero. Ressalta-se que o objetivo, nessa etapa, é apenas a mistura mecânica dos compostos EPDM, agentes de cura e a parafina.

$\mathrm{Na}$ amostra, na qual a parafina foi adicionada, antes do processo de reticulação, Amostra 3, observou-se a menor diminuição quanto ao alongamento na ruptura, demonstrando predomínio das características da fase elastomérica, presentes para essa propriedade, mas com aumento do Modulo de Young e da dureza, relacionados a uma maior rigidez do material, influenciadas pelo processo de reticulação realizado. Estudos realizados por Abu-Abdeen (2001) relatam que a parafina pode promover o isolamento das reticulações presentes na cadeia polimérica, as quais podem resultar em defeitos na rede cruzada da estrutura, influenciando as propriedades mecânicas do produto acabado.

$\mathrm{Na}$ inserção da parafina, após a reticulação, Amostra 4, estima-se que a parafina não se dispersou internamente no material, depositando-se na superfície do mesmo, influenciando na eficiência da incorporação e homogeneidade da mistura obtida, pois com a reticulação da fase elastomérica presente, promove-se uma maior resistência mecânica e rigidez, porém, dificulta-se a inserção e, consequentemente, homogeneização de outras substâncias adicionadas, durante a etapa.

Ainda, a presença dos grupos hidroxila $(-\mathrm{OH})$ e carboxila $(\mathrm{C}=\mathrm{O})$ na estrutura da parafina, utilizada neste estudo, podem favorecer ligações intracadeias fortes no material, justificando a sua influência no comportamento das propriedades mecânicas de tração, alongamento na ruptura e dureza. Essa afirmação é comprovada ao comparar as amostras presentes na tabela 6 , onde há uma diferença de aproximadamente 0,5 $\mathrm{MPa}$ para as Amostras 2, $3 \mathrm{e}$ 4, em relação à Amostra 1, demonstrando pequena fragilização das cadeias, as quais são reafirmadas pela simultânea diminuição de alongamento na ruptura de $80 \%$ das demais amostras, com exceção à Amostra 3 que demonstrou resultados mais característicos de um material flexível e resistente.

A partir dos resultados expostos, conclui-se que a presença da parafina na mistura TPV EPDM/ $\mathrm{PP}_{\text {homo }}$ tem influência nas características mecânicas do material, diminuindo os valores de propriedades de tensão de ruptura, alongamento e, por consequência, a tenacidade das amostras, ou seja, causando pequena fragilização de suas propriedades elastoméricas. A amostra que apresentou resultados mecânicos com menores perdas de alongamento e valor de tensão máxima intermediária foi representada pela pré-reticulação, sendo, então, essa etapa selecionada para a continuação do experimento. Quanto aos testes de tração e dureza Shore A, observa-se diferenças de resultados, em relação à etapa de introdução da parafina.

Para avaliar a concentração de parafina na mistura, foi mantida a quantidade de 2,5 phr do sistema de cura composto de Perkadox e TAC50. Esse valor foi previamente definido, demonstrado no tópico 3.3, como sendo de maior eficiência. A seguir, estão relacionados os resultados dos testes de tração e Shore A, para o estudo de quantificação da parafina na composição da mistura, tabela 7 .

Observa-se, a partir dos dados demonstrados na tabela 7, um comportamento semelhante entre as diferentes amostras com valores máximos de tensão em uma faixa de 4,3 a $5 \mathrm{MPa}$ e deformação total de 300 a $400 \%$. Quanto ao fator de alongamento na ruptura, observa-se que, embora se evidencie um aumento do valor nominal do alongamento na ruptura 
com o acréscimo do percentual inserido de parafina, os desvios obtidos foram altos, dificultando uma avaliação conclusiva sobre o efeito gerado. Quanto à rigidez e dureza, observa-se um aumento em ambos, conforme o aumento da parafina inserida na mistura $\mathrm{EPDM} / \mathrm{PP}_{\text {homo }}$. Supõe-se que o aumento desses fatores esteja relacionado à composição química da parafina utilizada, a qual contém substâncias, contendo grupos carbonila e hidroxila em sua estrutura, promovendo ligações intracadeias que influenciaram na propriedade do produto final, visto que o percentual do sistema de cura usado foi o mesmo para todas as amostras.

Tabela 7 - Teste de tração e dureza Shore A, para amostras de EPDM/PP homo 100/30, contendo parafina nas proporções 5,10,15, 20, 25 e $30 \mathrm{phr}$

\begin{tabular}{ccccc}
\hline $\begin{array}{c}\text { Amostra } \\
\text { (Parafina em phr) }\end{array}$ & $\begin{array}{c}\text { Tensão de } \\
\text { ruptura (MPa) }\end{array}$ & $\begin{array}{c}\text { Alongamento } \\
\text { na ruptura (\%) }\end{array}$ & $\begin{array}{c}\text { Módulo de } \\
\text { Young (MPa) }\end{array}$ & $\begin{array}{c}\text { Dureza } \\
\text { Shore A }\end{array}$ \\
\hline 5 & $4,6 \pm 0,5$ & $343,5 \pm 65,0$ & 0,17 & $77,0 \pm 1,3$ \\
10 & $5,0 \pm 0,3$ & $357,3 \pm 24,8$ & 0,21 & $79,5 \pm 1,7$ \\
15 & $4,5 \pm 0,3$ & $330,5 \pm 46,7$ & 0,20 & $77,8 \pm 0,7$ \\
20 & $4,7 \pm 0,3$ & $360,2 \pm 48,1$ & 0,21 & $80,7 \pm 0,5$ \\
25 & $4,6 \pm 0,2$ & $387,2 \pm 27,6$ & 0,21 & $80,5 \pm 0,8$ \\
30 & $4,3 \pm 0,2$ & $392,9 \pm 25,5$ & 0,23 & $83,2 \pm 0,7$ \\
\hline
\end{tabular}

Fonte: Os autores (2019).

Os dados analisados não demonstraram variações expressivas na tensão de ruptura, como observado para as misturas de referência, demonstradas no tópico 3.2. Supõe-se que a causa da diferenciação, em relação à amostra EPDM + Parafina é dada, pois a amostra já passou por reticulação, logo, o fenômeno de aumento de tensão de ruptura, observado na amostra EPDM + Parafina por movimento das cadeias de carbono, para formação de zonas amorfas regulares, não ocorre (ABU-ABDEEN, 2001).
Portanto, o gradual acréscimo na proporção da parafina na composição da amostra demonstrou pequena influência no conjunto das propriedades mecânicas, mantendo o comportamento do TPV EPDM/ $\mathrm{PP}_{\text {homo }} /$ Agentes de cura, sem grandes variações quanto às suas propriedades mecânicas.

A seguir, estão relacionados os resultados obtidos nos ensaios mecânicos para as diferentes etapas do estudo de amostras de referência, incorporação e quantificação da parafina, tabela 8.

Tabela 8 - Relação de resultados dos ensaios mecânicos, realizados para os diferentes estudos realizados

\begin{tabular}{cccccc}
\hline Séries & Amostra & $\begin{array}{c}\text { Tensão de } \\
\text { ruptura (MPa) }\end{array}$ & $\begin{array}{c}\text { Alongamento } \\
\text { na ruptura (\%) }\end{array}$ & $\begin{array}{c}\text { Módulo de } \\
\text { Young (MPa) }\end{array}$ & $\begin{array}{c}\text { Dureza } \\
\text { Shore A }\end{array}$ \\
\hline \multirow{3}{*}{ Referência } & EPDM Puro & $1,1 \pm 0,0$ & $945,3 \pm 23,2$ & 0,02 & $21,3 \pm 0,8$ \\
& EPDM + PP homo & $3,5 \pm 0,2$ & $481,1 \pm 36,1$ & 0,11 & $64,8 \pm 1,6$ \\
EPDM + Parafina & $5,5 \pm 0,7$ & $884,0 \pm 18,8$ & 0,10 & $51,0 \pm 3,0$ \\
Amostra & Pré-batelada & $5,0 \pm 0,3$ & $294,5 \pm 20,1$ & 0,22 & $80,0 \pm 0,6$ \\
10 phr & Pré-reticulação & $5,0 \pm 0,3$ & $357,3 \pm 24,8$ & 0,21 & $79,5 \pm 1,6$ \\
Parafina & Pós-reticulação & $4,7 \pm 0,2$ & $291,0 \pm 24,1$ & 0,19 & $78,8 \pm 1,2$ \\
& 5 phr Parafina & $4,6 \pm 0,5$ & $343,5 \pm 65,0$ & 0,17 & $77,0 \pm 1,3$ \\
& 10 phr Parafina & $5,0 \pm 0,3$ & $357,3 \pm 24,8$ & 0,21 & $79,5 \pm 1,7$ \\
Percentual & 15 phr Parafina & $4,5 \pm 0,3$ & $330,5 \pm 46,7$ & 0,20 & $77,8 \pm 0,7$ \\
Parafinas & 20 phr Parafina & $4,7 \pm 0,3$ & $360,2 \pm 48,1$ & 0,21 & $80,7 \pm 0,5$ \\
& 25 phr Parafina & $4,6 \pm 0,2$ & $387,2 \pm 27,6$ & 0,21 & $80,5 \pm 0,8$ \\
& 30 phr Parafina & $4,3 \pm 0,2$ & $392,9 \pm 25,5$ & 0,23 & $83,2 \pm 0,7$ \\
\hline
\end{tabular}

Fonte: Os autores (2019).

Observa-se, a partir da tabela 8, que para a tensão de ruptura, a partir da incorporação da parafina na amostra TPV EPDM/PP ${ }_{\text {homo }}$, houve pequena variação quanto a sua etapa de inserção no processo, sendo 
essa de aproximadamente $0,3 \mathrm{MPa}$, e o seu gradual aumento na amostra influenciou de forma a diminuir esse valor em aproximadamente $0,7 \mathrm{MPa}$.

A amostra produzida tem como intenção o recobrimento de superfícies as quais necessitam de temperaturas, acima de $40^{\circ} \mathrm{C}$ e abaixo de $180^{\circ} \mathrm{C}$, para seu funcionamento, a faixa de temperatura é destacada, devido às temperaturas de fusão parcial da parafina e degradação do material, respectivamente. A característica de armazenamento de temperatura de um material PCM pode resultar na diminuição de custo energético para equipamentos ao manter temperaturas intermediárias por períodos mais longos, através da energia armazenada, proveniente do calor latente do PCM. O material, em estudo, deve apresentar boa flexibilidade e alta resistência à tração, para adequar-se a diversos usos e boa superfície de contato com os equipamentos.

Os resultados mecânicos obtidos demonstraram as características desejadas, pois a utilização da parafina em crescente quantidade não causou brusca fragilização das características mecânicas iniciais de TPV na amostra, levando a entender que ainda há possibilidade para maiores quantidades de material PCM a ser inserido e, logo, maior atuação e potencialidade térmica para o material obtido.

\subsection{Inchamento}

Para o estudo da análise de inchamento das amostras obtidas, o grau de inchamento (Q), assim como os valores de massa inicial da amostra e massa do solvente absorvido estão descritos na tabela 9. Ressalta-se que ao final do teste, foram encontrados resíduos com aspecto de óleo no fundo do recipiente utilizado para realização do teste de inchamento provenientes, provavelmente, do componente parafina inserido na mistura. Descartou-se o óleo de fabricação EPDM, pois o elastômero usado, no presente estudo, foi fabricado livre de óleo em sua composição. Assim, supõem-se que ocorreu remoção parcial da parafina, presente nas amostras estudadas, durante o experimento. Ressaltase que as amostras utilizadas para a referida análise foram reticuladas com 2,5 phr de agentes de cura Perkadox 1440B e TAC50\%, e a análise foi realizada na temperatura de $23^{\circ} \mathrm{C}$ em banho termostatizado.

Tabela 9 - Massas obtidas e grau de inchamento no solvente ciclohexano

\begin{tabular}{ccc}
\hline Amostra & Grau de inchamento $(\mathbf{Q})$ & $\mathbf{V r}$ \\
\hline $\mathrm{EPDM} / \mathrm{PP}_{\text {homo }} 100 / 30$ com 5 phr parafina & 1,769 & 0,565 \\
$\mathrm{EPDM} / \mathrm{PP}_{\text {homo }} 100 / 30$ com 10 phr parafina & 1,725 & 0,580 \\
$\mathrm{EPDM} / \mathrm{PP}_{\text {homo }} 100 / 30$ com 15 phr parafina & 1,777 & 0,563 \\
$\mathrm{EPDM} / \mathrm{PP}_{\text {homo }} 100 / 30$ com 20 phr parafina & 1,781 & 0,561 \\
$\mathrm{EPDM} / \mathrm{PP}_{\text {homo }} 100 / 30$ com 25 phr parafina & 1,836 & 0,545 \\
$\mathrm{EPDM} / \mathrm{PP}_{\text {homo }} 100 / 30$ com 30 phr parafina & 1,959 & 0,510 \\
\hline
\end{tabular}

Fonte: Os autores (2019).

Com os dados de grau de inchamento, demonstrados na tabela 9, percebe-se que houve mudança percentual de 5-10\% no grau de inchamento nas amostras com 25 e $30 \mathrm{phr}$ de parafina inseridas. A partir desses dados, pode-se inferir que a inserção da parafina, em maiores quantidades na amostra, pode induzir a uma expansão de volume dentro das cadeias reticuladas, criando mais espaços livres para a entrada do solvente.

\subsection{Infravermelho}

Para avaliar o material residual da etapa de extração da fase termoplástica $\left(\mathrm{PP}_{\text {homo }}\right)$ da amostra EPDM/ $\mathrm{PP}_{\text {homo }} /$ Parafina, utilizando xileno quente, a análise por espectrometria de infravermelhos foi realizada, utilizando o acessório de reflexão atenuada total (ATR). A figura 3 apresenta a análise dos espectros ATR-FTIR realizada, utilizando o material residual do processo de extração das amostras de $\mathrm{EPDM} / \mathrm{PP}_{\text {homo }} /$ Parafina, contendo 5,10,15,20,25 e $30 \mathrm{phr}$ de parafina, das quais se observaram resíduos na parede e, no fundo do recipiente, onde a extração foi realizada.

Observa-se, pela figura 3(a), bandas de absorção na região entre $1300 \mathrm{~cm}^{-1}$ e $1000 \mathrm{~cm}^{-1}$, caracterizando a presença de grupos funcionais éter, os quais fazem parte da composição da parafina utilizada neste estudo já, na figura 3(b), são encontrados apenas bandas de absorção características de alcanos, também evidenciadas na figura 3(a). Logo, pode-se supor que no banho de xileno para remoção de $\mathrm{PP}_{\text {homo, }}$ houve 
também remoção parcial de parafina. Observa-se, ainda, em ambos os espectros, a presença de bandas na faixa $3000 \mathrm{~cm}^{-1} \mathrm{e} 2840 \mathrm{~cm}^{-1} \mathrm{e}$, posteriormente, bandas em $1450 \mathrm{~cm}^{-1}$ e $1465 \mathrm{~cm}^{-1}$, características de grupos $-\mathrm{CH}_{3} \mathrm{e}-\mathrm{CH}_{2}$ de alcanos, respectivamente, presente tanto no polipropileno quanto na parafina, comprovando a eficiência da extração do componente termoplástico da mistura. Resultados semelhantes foram obtidos por Lopes e Fascio (2004), corroborando com os evidenciados neste estudo.

Figura 3 - Análise ATR-FTIR das amostras na (a) parede e (b) fundo do recipiente

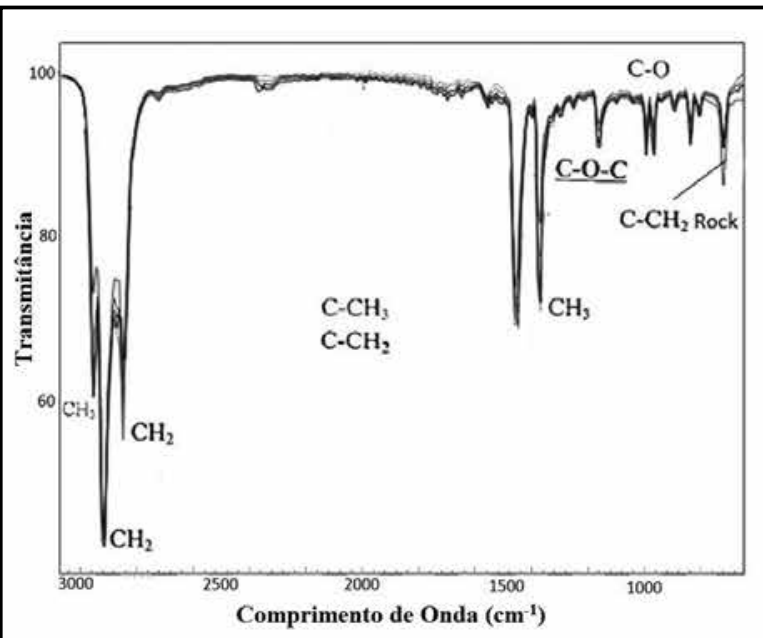

(a)

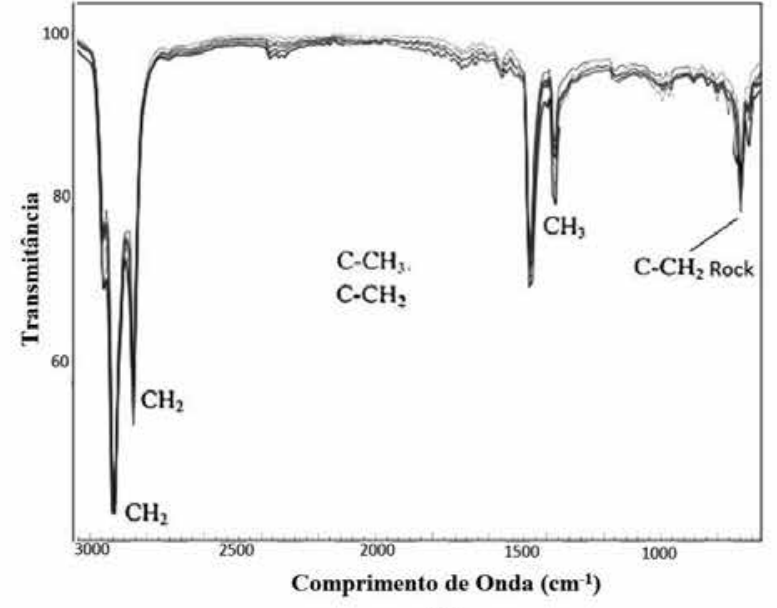

(b)

Fonte: Os autores (2019).

\subsection{Morfologia}

Para avaliar a morfologia das amostras TPV $\mathrm{EPDM} / \mathrm{PP}_{\text {homo }} /$ Parafina, obtidas no presente estudo, foi utilizada microscopia eletrônica de varredura (MEV). As amostras foram preparadas anteriormente, com a extração da fase termoplástica $\mathrm{PP}_{\text {homo }}$ em xileno a quente, com o intuito de observar vazios na estrutura os quais deveriam pertencer ao $\mathrm{PP}_{\text {homo }}$ removido, porém, conforme descrito no item 3.6, observou-se na análise de ATR-FTIR a presença de grupos funcionais, pertinentes à estrutura da parafina, fator que pode alterar o resultado previsto. A figura 4 apresenta as imagens obtidas, a partir da análise por microscopia eletrônica de varredura das amostras $\mathrm{EPDM} / \mathrm{PP}_{\text {homo }} /$ Parafina, com 5 a 30 phr de parafina, inserida à mistura.

É possível observar a presença de vazios de profundidade superficial nas amostras (a), (b), (e) e (f) analisadas, o que demonstra que a fase termoplástica foi parcialmente retirada, porém o processo de extração não foi eficiente como desejado. $O$ fato foi descrito no tópico 3.6 onde, após o processo de extração em solvente, realizou-se a análise de infravermelho, observando-se a presença de grupos funcionais, referentes à presença tanto de parafina quanto de $\mathrm{PP}_{\text {homo }}$ no extrato.
$\mathrm{Na}$ amostra com $10 \mathrm{phr}$ de parafina, representada por (b), há uma suposta boa extração do termoplástico, com boa demonstração de domínios de borracha na imagem, o que contrasta com a imagem, contendo 25 phr de parafina, onde há uma fase sobrepondo a amostra, podendo ser uma fração da parafina pura que se distribuiu sobre a superfície da amostra.

As amostras, contendo 15 e $20 \mathrm{phr}$ de parafina (c e d), apresentaram baixa eficiência para a extração do termoplástico que pode ser implicada, devido à mistura inferior dos componentes em relação às demais e, assim, a não absorção da parafina na mistura TPV. Já as imagens (a), (b) e (f) apresentaram características de fase co-contínua, através de uma boa mistura, resultando em uma extração mais eficiente.

A amostra com 25 phr (e) demonstrou boa elongação com menores desvios, preservando a tensão de ruptura, módulo de Young e dureza das demais proporções analisadas $(387,2 \pm 27,6 \% ; 4,6 \pm 0,2 \mathrm{MPa}$; 0,21 e $80,5 \pm 0,8$, respectivamente). Sendo assim, supõe-se que houve melhor inserção da parafina na composição da mistura e, a partir da imagem (e), encontram-se áreas com boa extração do componente termoplástico para comprovar essa afirmação.

Estudos de Song et al. (2010) obtiveram resultados semelhantes aos encontrados neste estudo, ao 
relacionar misturas de EPDM/Parafina e nano hidróxido de magnésio. Em seu estudo, descreve que a morfologia observada por microscopia eletrônica tem relação com a estrutura da rede tridimensional obtida, na qual a parafina se dispersa de maneira uniforme, pois é um composto homólogo ao EPDM, sendo facilmente misturada com o elastômero, explicitando o princípio de solubilidade "semelhante dissolve semelhante".

Figura 4 - Microscopia eletrônica de varredura de amostras de EPDM/PP homo $100 / 30$, contendo 5 (a), 10 (b), 15 (c), 20 (d), 25 (e) e 30 (f) phr de parafina

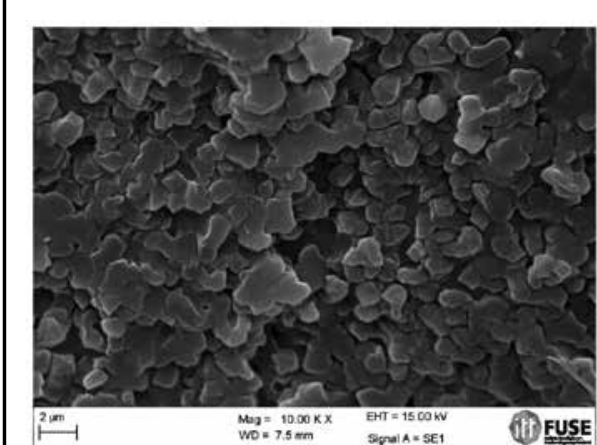

(a)

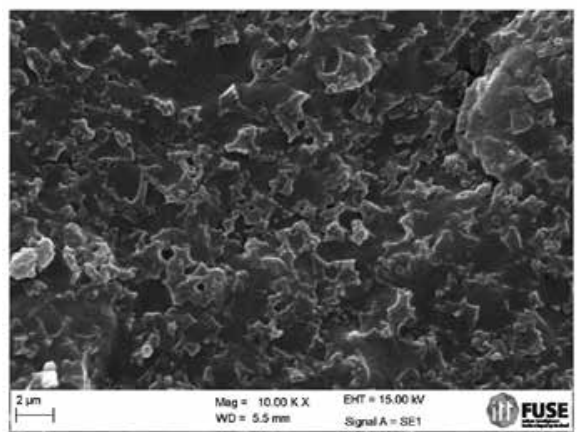

(c)

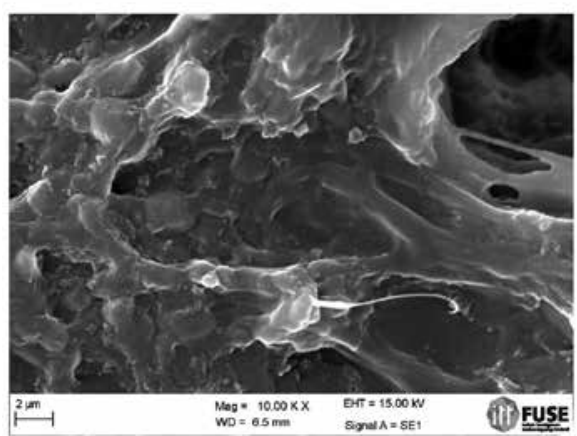

(e)

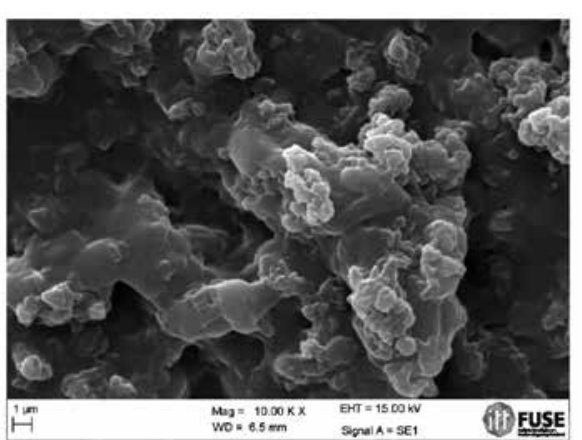

(b)

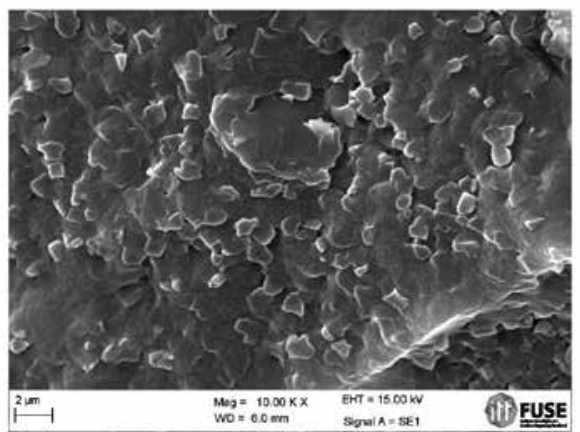

(d)

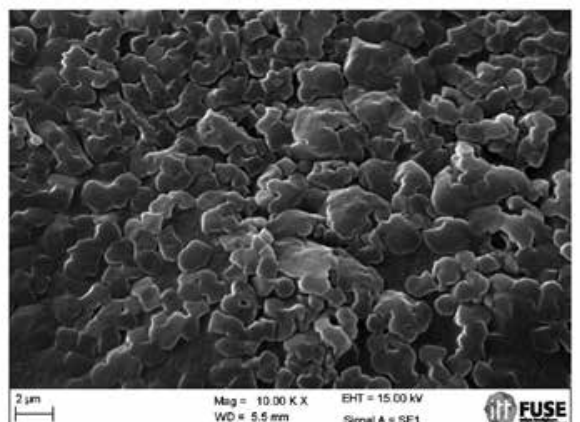

(f)

Fonte: Os autores (2019).

A baixa eficiência no processo de extração termoplástica de $\mathrm{PP}_{\text {homo }}$ causa complicações na análise por microscopia eletrônica de varredura associadas à compatibilidade da parafina com EPDM, uma vez que, pode causar mascaramento e/ou dificultar uma boa observação da fase formada. Uma solução para o problema é investigar diferentes processos de extração, com solventes compatíveis com parafina, sem danificar a amostra de TPV, facilitando o processo de observação.

Para as amostras, contendo 5,20,25 e $30 \mathrm{phr}$ de parafina, observa-se uma fase co-contínua, a qual 
supõe-se que houve extração pouco eficiente da fase $\mathrm{PP}_{\text {homo }}$ na amostra, pois evidencia-se espaços presentes.

\section{Conclusões}

A partir da premissa de investigar a matéria-prima parafina, visando sua futura aplicação como material de troca de fase (PCM), investigou-se a obtenção de material TPV contendo uma mistura de $\mathrm{EPDM} / \mathrm{PP}_{\text {homo }}$, avaliando-se seu comportamento mecânico, após a inserção do componente parafina, assim como, as etapas de inserção dela e possível influência quanto ao comportamento de expansão do produto acabado obtido.

Em relação aos ensaios mecânicos, as amostras estudadas, inserindo $2,5 \mathrm{phr}$ de agentes de cura e 30 phr de parafina, demonstraram as melhores características para um material versátil, para aplicações de recobrimento de equipamentos, necessitando de considerável flexibilidade e resistência mecânica, com tensão de ruptura de 4,3 $\pm 0,2 \mathrm{MPa}$ e alongamento na ruptura de $392,9 \pm 25,5 \%$, sendo esse parâmetro o maior alongamento observado, ao serem comparados com as demais amostras obtidas.

$\mathrm{Na}$ análise de inchamento, observa-se que, com maiores quantidades do PCM inserido, principalmente nas amostras, contendo 25 e $30 \mathrm{phr}$ de parafina, houve uma maior variação do grau de inchamento a $25^{\circ} \mathrm{C}$ e, a partir disso, entende-se que o crescente aumento de parafina na composição da mistura tem, como consequência, a expansão de volume no interior das cadeias reticuladas. Para fins práticos de aplicação como material de recobrimento, essa característica necessita de maior investigação, para determinar se esse fator pode atuar na versatilidade do material como TPV com características de PCM.

Durante a preparação da amostra para a análise por MEV, observou-se a presença de material residual cuja análise por FTIR-ATR confirmou a presença de PP e parafina no recipiente, contendo o material residual da extração realizada. Sendo assim, conclui-se que o processo de extração da fase $\mathrm{PP}$ foi eficaz, porém não eficiente. Nas micrografias obtidas por MEV, é possível observar que a inserção da parafina influenciou na morfologia obtida, onde se observa um recobrimento, à medida que aumenta o percentual de parafina inserido.

Ressalta-se que a intenção, para o estudo do material TPV com acréscimo de materiais PCM, é desenvolver um material versátil o qual pode atuar como recobrimento de superfícies os quais necessitam de temperaturas, acima de $40^{\circ} \mathrm{C}$ e abaixo de $180^{\circ} \mathrm{C}$, para seu funcionamento. Destacou-se, neste estudo, os resultados obtidos da amostra EPDM/PP homo $100 / 30$, contendo 2,5 phr de agentes de cura (Perkadox 1440B e TAC50\%) e com 30 phr de parafina, sendo adicionada na etapa anterior à reticulação (pré-reticulação), pois a mesma apresentou os melhores valores mecânicos e potencial, para o uso prático.

\section{Referências}

ABU-ABDEEN, M. Degradation of the mechanical properties of composite vulcanizates loaded with paraffin wax. Journal of Applied Polymer Science, v. 81, n. 9, p. 2265-2270, 2001.

BANNO, A. et al. Pyrolysis behavior of a paraffin-based thermoplastic polymer used hybrid rocket fuel. International Journal of Energetic Materials and Chemical Propulsion, v. 18, p. 341-354, 2019. DOI: 10.1615/ IntJEnergeticMaterialsChemProp.2019028195.

BOIOCCHI, M. et al. A wide characterization of paraffin-based fuels mixed with styrene-base thermoplastic polymers for hybrid propulsion. Progress in Propulsion Physics, v. 8, p. 241-262, 2016. DOI:10.1051/eucass/201608241.

CHATTERJEE, T. et al. Novel thermoplastic vulcanizates (TPVs) based on silicone rubber and polyamide exploring peroxide cross-linking. eXPRESS Polymer Letters, v. 8, n. 4, p. 220-231, 2014. DOI:10.3144/expresspolymlett.2014.26.

CHEN, P. et al. Metal foam embedded in SEBS/ paraffin/HDPE form-stable PCMs for thermal energy storage. Solar Energy Materials and Solar Cells, v. 149, p, 60-65, 2016. DOI:10.1016/j.solmat.2015.12.041.

$\mathrm{CHO}, \mathrm{H}$. et al. Deformation mechanisms of thermoplastic elastomers: stress-strain behavior and constitutive modeling. Polymer, v. 128, n. 5, p. 87-99, 2017. DOI:10.1016/j.polymer.2017.08.065.

CORAN, A. Y. Vulcanization: conventional and dynamic. Rubber Chemistry and Technology, v. 68, n. 3, p. 351-375, 1995. DOI: 10.5254/1.3538748.

ELLUL, D. M.; TSOU, A. H.; HU, W. Crosslink densities and phase morphologies in thermoplastc vulcanizate. Polymer, v. 45, n. 10, p. 3351-3358, 2004. DOI:10.1016/j.polymer.2004.03.029.

GULFAM, R.; ZHANG, P.; MENG, Z. Advanced thermal systems driven by paraffin-based phase change materials. Applied Energy, v. 238, p. 582-611, 
2019. DOI: 10.1016/j.apenergy.2019.01.114.

HOLDEN, G.; KRICHELDORF, H. R.; QUIRK, R. P. Thermoplastic elastomers. 3th ed. Cincinnati: Hanser Gardner, 2004.

IMATO, K. et al. Repeatable mechanochemical activation of dynamic covalent bonds in thermoplastic elastomers. Chemical Communications, n. 52, p. 10482-10485, 2016. DOI: 10.1039/C6CC04767J.

KHIMI, S. R.; PICKERING, K. L. A new method to predict optimum cure time of rubber compound using dynamic mechanical analysis. Journal of Applied Polymer Science, v. 131, n. 6, 2014. DOI: 10.1002/app.40008.

KUMAR, T. R. S.; JEGADHEESWARAN, S.; CHANDRAMOHAN, P. Performance investigation on fin type solar still with paraffin wax as energy storage media. Jornal of Thermal Analysis and Calorimetry, v. 136 , n. 2, p. 101-112, 2018. DOI:10.1007/s10973018-7882-7.

LOPES, A. W.; FASCIO, M. Esquema para interpretação de espectros de substâncias orgânicas na região do infravermelho. Química Nova, v. 27 , n. 4, p. 670-673, 2004. DOI: 10.1590/S010040422004000400025 .

LUO, Z. et al. Numerical and experimental study on temperature control of solar panels with form-stable paraffin/expanded graphite composite PCM. Energy Conversion and Management, v. 149, p. 416-423, 2017. DOI:10.1016/j.enconman.2017.07.046.

MATEI, E. et al. Recycled polypropylene improved with thermoplastic elastomers. International Journal of Polymer Science, p. 1-10, 2017. DOI: 10.1155/2017/7525923.
MCMULLIN, E.; REBAR, H. T.; MATHER, P. T. Biodegradable thermoplastic elastomers incorporation POSS: synthesis, microstructure, and mechanical properties. Macromolecules, v. 49 , n. 10 , p. $3769-3779,2016$. DOI:10.1021/acs. macromol.6b00470.

NAKASON, C. et al. Dynamic vulcanization of natural rubber/high-density polyethylene blends: Effect of compatibilization, blend ratio and curing system. Polymer Testing, v. 25, n. 6, p. 782-796, 2006. DOI: $10.1016 /$ j.polymertesting.2006.05.001.

NING, N. et al. Preparation, microstructure, and microstructure-properties relationship of thermoplastic vulcanizates (TPVs): a review. Progress in Polymer Science, v. 79, p. 61-97, 2018. DOI: 10.1016/j.progpolymsci.2017.11.003.

RAMAKRISHNAN, S. et al. A novel paraffin/ expanded perlite composite phase change material for prevention of PCM leakage in cementitious composites. Applied Energy, v. 157, p. 85-94, 2015. DOI:10.1016/j.apenergy.2015.08.019.

SONG, G. et al. Preparation and characterization of flame retardant form-stable phase change materials composed by EPDM, paraffin and nano magnesium hydroxide. Energy, v. 35, n. 5, p. 2179-2183, 2010. DOI:10.1016/j.energy.2010.02.002.

WATTS, A.; KUROKAWA, N.; HILLMYER, M. A. Strong, resilient, and sustainable aliphatic polyester thermoplastic elastomers. Biomacromolecules, v. 18 , n. 6 , p. $1845-1854,2017$. DOI:10.1021/acs. biomac.7b00283.

YU, K. et al. Mechanics of self-healing thermoplastic elastomers. Journal of the Mechanics and Physics of Solids, v. 137, 2020. DOI: 10.1016/j.jmps.2019.103831. 
